\title{
The crazy-paving pattern on computed tomography
}

\author{
Nimrod Maimon MD, Dov Heimer MD
}

Previously published at www.cmaj.ca

$\mathrm{A}$ $\mathrm{n}$ otherwise healthy 46-year-old man presented with a six-month history of exercise-related shortness of breath and dry cough. His oxygen saturation was $86 \%$ on room air. Testing of pulmonary function showed a moderately restrictive ventilatory defect, with diffusion capacity of $45 \%$ of the predicted values. A computed tomographic scan of the chest showed diffuse ground-glass attenuation, superimposed on a network of interlobular septal thickening and intralobular lines, consistent with the crazypaving pattern (Figure 1).

The crazy-paving pattern found on computed tomography of the lungs is a nonspecific radiologic sign named after the appearance of paths made with pieces of stone or concrete. It was first described in the late 1980s in patients with pulmonary alveolar proteinosis, but has now been described in association with at least 15 pulmonary diseases. ${ }^{1}$ Histologically, three processes lead to the crazy-paving pattern: an alveolar filling process, interstitial fibrosis and a combination of the two. The lung insults associated with these processes can be related to infection, neoplasm, inhalation, toxins and fluid overload, and can also be idiopathic. ${ }^{2}$

In our patient, fluid from bronchoalveolar lavage had a milky appearance and contained macrophages that tested positive on periodic acid-Schiff staining, consistent with the diagnosis of idiopathic pulmonary alveolar proteinosis. He underwent whole-lung lavage under general anesthesia, which involves removal of the lipoproteinaceous material by repeated dilution with saline. The patient's symptoms were temporarily relieved. He has undergone this procedure on average every two years for the past six years. Four years ago, a trial of therapy with granulocyte-macrophage colony-stimulating factor did not lead to clinical or radiologic improvement.

Little is known about the natural course or optimal treatment of idiopathic pulmonary alveolar proteinosis, although

From the Division of Respirology, Department of Medicine, University of Toronto, and Toronto General Research Institute, University Health Network (Maimon), Toronto, Ont., the Division of Pulmonology, Department of Medicine, Ben-Gurion University of the Negev, and Soroka University Medical Center (Heimer), Beer-Sheva, Israel

CMAJ 2010. DOI:10.1503/cmaj.091422

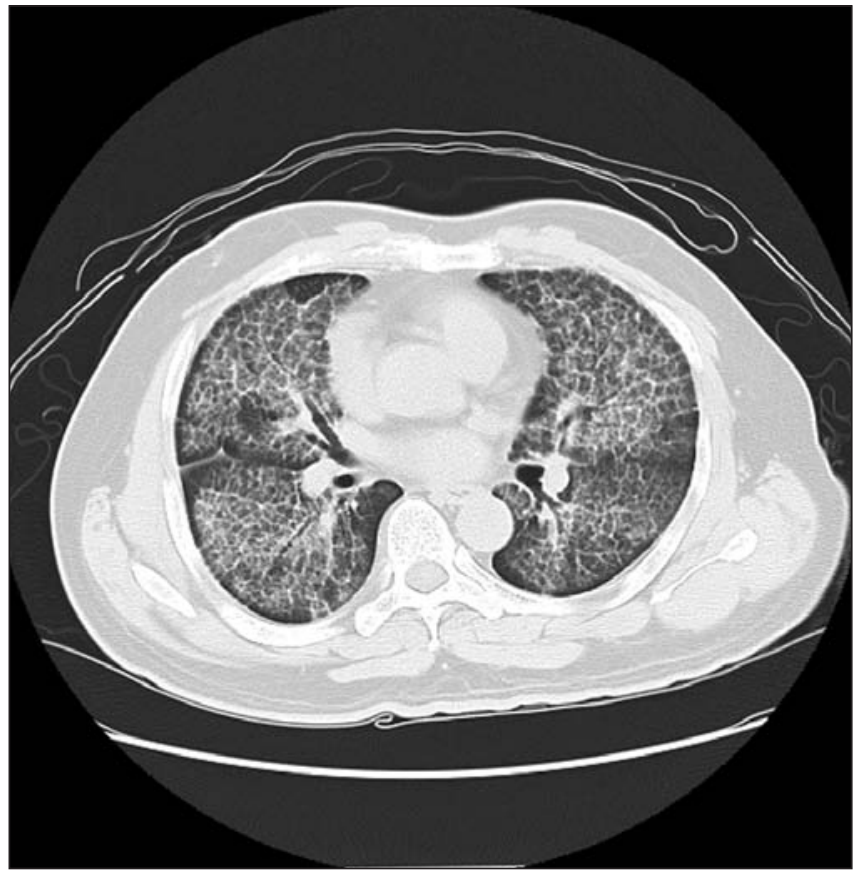

Figure 1: Computed tomographic scan of the chest of a 46year-old man showing the crazy-paving pattern.

most patients will have symptomatic relief following pulmonary lavage. ${ }^{3}$ In some patients, the disease is treated with lung transplantation. Because of the uncertainty surrounding our patient's prognosis, we are providing long-term follow-up at our chest clinic.

Competing interests: None declared.

\section{REFERENCES}

1. Rossi SE, Erasmus JJ, Volpacchio M, et al. "Crazy-paving" pattern at thinsection CT of the lungs: radiologic-pathologic overview. Radiographics 2003; 23:1509-19.

2. Johkoh T, Itoh H, Müller NL, et al. Crazy-paving appearance at thin-section CT: spectrum of disease and pathologic findings. Radiology 1999;211:155-60.

3. Venkateshiah SB, Yan TD, Bonfield TL, et al. An open-label trial of granulocyte macrophage colony stimulating factor therapy for moderate symptomatic pulmonary alveolar proteinosis. Chest 2006;130:227-37. 